\title{
Targeted Land-Cover Classification
}

\author{
Diego Fernàndez-Prieto and Mattia Marconcini \\ European Space Agency, ESA-ESRIN, Earth Observation Science, Applications and \\ Future Technologies Department, Frascati, Italy; \{diego.fernandez, mattia.marconcini\}@esa.int
}

\begin{abstract}
In several real-world applications the objective of land-cover classification is actually limited to map one or few specific "targeted" land-cover classes over a certain area. In such cases, ground-truth information is generally available for the only land-cover classes of interest, which limits (or hinders) the possibility of successfully employing standard supervised approaches that require an exhaustive ground truth for all the land-cover classes characterizing the investigated area. In this paper, we present a novel technique capable of addressing this challenging issue (formulated in terms of a compound decision problem) by exploiting the only ground truth available for the targeted land-cover classes. In particular, the proposed method jointly exploits the Expectation-Maximization (EM) algorithm and an iterative labeling strategy based on Markov random fields (MRF) accounting for spatial correlation. Experimental results on different datasets confirmed the effectiveness and the reliability of the proposed technique, which provided classification accuracies comparable with those obtained by fully-supervised methods.
\end{abstract}

Keywords. land-cover mapping, targeted classification, Expectation maximization, Markov random fields.

\section{Introduction}

In several application domains (e.g., agriculture, forestry, spatial planning, ecosystem monitoring, disaster management, habitat mapping, etc), the ultimate operational objective of land-cover classification is actually limited to the identification of only one or few specific land-cover classes of interest (i.e., "targeted classes"), disregarding all the other potential classes present in the area under analysis, which could be completely unknown to an operator.

This type of problems (hereinafter referred to as "targeted land-cover classification") could be effectively solved by traditional supervised techniques provided that full and exhaustive groundtruth information (including samples from all the land-cover classes characterizing the area of interest) is available. Nevertheless, such a requirement is seldom satisfied and presents several practical drawbacks and limitations, both in terms of time and economic cost that may render this task difficult to achieve in most real-life cases.

However, the possibility of performing an effective targeted classification only using groundtruth samples available for the classes of interest would represent a significant advantage. Indeed, the collection of labeled samples for a single or few specific classes is certainly simpler and cheaper (e.g., a trained operator could even accomplish it by photo-interpretation). In this paper, we address such a challenging issue and propose a novel technique capable of solving targeted classification problems by exploiting the ground truth available only for the classes of interest, while providing accuracies comparable with those of traditional fully-supervised methods.

In this paper, we formulate this complex issue in terms of a compound decision problem [1] and propose a novel targeted classification (TC) technique capable of exploiting the only prior knowledge available for the specific land-cover classes of interest (thus avoiding the need to rely on exhaustive ground-truth information for all the classes), while providing accuracies comparable with those of fully-supervised methods. 
The proposed method aims at estimating the probability density function (PDF) and the prior of both the class(es) of interest and the remaining unknown land-cover classes (for which no ground truth is available) represented as a single unknown information class. In particular, PDFs are approximated by a mixture of suitable basis functions whose free parameters are determined employing the iterative Expectation-Maximization (EM) algorithm [2]. Pixels belonging to the land-cover classes of interest are then identified using an iterative labeling strategy based on Markov random fields (MRF) [3] which allows taking into account spatial correlation, as well as properly constraining the probability estimates.

An extensive experimental analysis and cross-comparisons with both state-of-the-art fully supervised support vector machines (SVM) [4], as well as with ensembles of support vector data description (SVDD) [5] classifiers (which are specifically designed for handling the particular case of a single class of interest) on different datasets confirmed the effectiveness and the reliability of the proposed technique.

\section{Problem formulation and assumptions}

Let us consider a $I \times J$ remote-sensing image $\mathrm{X}=\left\{\mathbf{x}_{i j}\right\}_{i, j=1}^{I, J}, \mathbf{x}_{i j} \in \square^{D}$, where $\mathbf{x}_{i j}$ represents the $D$ dimensional feature vector associated to the pixel at position $(i, j)$ (even derived from different sets of sensors and merged using a stacked vector approach [6]).

Let $\Omega=\left\{\omega_{1}, \ldots, \omega_{L}\right\}$ be the set of land-cover classes characterizing $X$. In the following, we will denote as $\left\{\omega_{\text {ntt }}, \ldots, \omega_{\text {nts }}\right\} \in \Omega, S<L$, the information classes of interest for which $N_{1}, \ldots, N_{S}$ labeled training patters are available, respectively. Hence, $\omega_{\mathrm{nk}}=\Omega-\left\{\omega_{\mathrm{ntl}}, \ldots, \omega_{\mathrm{nts}}\right\}$ will represent the corresponding unknown class (consisting of the merger of remaining classes for which no ground truth is available).

Let $\mathrm{C}=\left\{C_{i j}\right\}_{i, j=1}^{I, J}$ denote a set of labels for $\mathrm{X}$, where $C_{i j} \in\left\{\omega_{\mathrm{ntt}}, \ldots, \omega_{\mathrm{nts}}, \omega_{\mathrm{nnk}}\right\}$ is associated to the pixel at position $(i, j)$. In this framework, our aim is to identify the set $\tilde{\mathrm{C}}$ maximizing the posterior probability given the image $X$ and, finally, to draw pixels solely belonging to the targeted landcover classes of interest $\omega_{\mathrm{ntt}}, \ldots, \omega_{\mathrm{nts}}$. This can be formalized as a compound decision problem [1]:

$$
\tilde{\mathrm{C}}=\underset{\mathrm{C}}{\operatorname{argmax}}\{P(\mathrm{C} \mid \mathrm{X})\}
$$

According to the Bayes theory, finding a solution to (1) is equal to determine the sets of labels maximizing the likelihood $\mathrm{L}(\mathrm{X} \mid \mathrm{C})$ :

$$
\tilde{\mathrm{C}}=\underset{\mathrm{C}}{\operatorname{argmax}}\{\mathrm{L}(\mathrm{X} \mid \mathrm{C})=P(\mathrm{C}) \cdot p(\mathrm{X} \mid \mathrm{C})\}
$$

where $p(\mathrm{X} \mid \mathrm{C})$ represents the conditional PDF.

\section{Proposed targeted classification technique}

For addressing the complex task described in Section 2, we propose a novel technique aimed at approximating the class-conditional density $p(\mathrm{X} \mid \mathrm{C})$ as a mixture of suitable basis kernel functions and estimating the prior probability $P(\mathrm{C})$ properly taking into consideration the spatial context.

The rationale is based on the observation that the PDF of an image can be always approximated by a mixture of suitable kernels (i.e., Parzen density estimation [1]). Accordingly, similarly to what is commonly done in the context of Radial Basis Function Neural Networks (RBF-NN) [7], we model for each pixel the PDF $p\left(\mathbf{x}_{i j}\right)$ as a mixture of $K$ circularly symmetric multivariate Gaussian functions. Kernel parameters (i.e., centers and variances) are initialized using the $k$-mean clustering 
algorithm [7], whereas final estimates are obtained by using the EM algorithm [2]. Then, classconditional densities of the classes of interest $\left\{p\left(\mathbf{x}_{i j} \mid \omega_{\text {nts } s}\right)\right\}_{s=1}^{S}$ are modeled by properly weighting the resulting set of kernels using again the EM algorithm over the only training samples available for the corresponding $s$-th class of interest $\omega_{\text {nts }}$. This is somewhat analogous to the training phase of RBF-NN which is generally carried out in two steps: i) selection of centers and variances of the kernel functions associated to hidden units on the basis of clustering techniques; and ii) computation of weights associated to the connections between the hidden and output layers on the basis of available training patterns.

The PDF of the entire image is itself a mixture of the conditional densities of both the classes of interest and the unknown class weighted by corresponding prior probabilities. Accordingly, we obtain a first rough approximation for $p\left(\mathbf{x}_{i j} \mid \omega_{\text {unk }}\right)$ initializing priors to $1 /(S+1)$ (since we consider $S$ classes of interest plus the unknown class). Afterwards, estimates are refined using MRFs accounting for spatial correlation, which permits to model $P\left(C_{i j}\right)$ considering the local neighborhood of each pixel. Finally, pixels belonging to the targeted land-cover classes of interest are identified by minimizing a proper energy function. In the following, we will first introduce the method adopted for modeling both the class-conditional densities and the prior probability; then, we will present the iterative strategy for identifying the sets of labels $\tilde{\mathrm{C}}$ maximizing the likelihood $\mathrm{L}(\mathrm{X} \mid \mathrm{C})$.

\subsection{Conditional density modeling}

Computing $p(\mathrm{X} \mid \mathrm{C})$ requires the estimation of the class-conditional densities $\left\{p\left(\mathbf{x}_{i j} \mid \omega_{\mathrm{nts} s}\right)\right\}_{s=1}^{S}$, and $p\left(\mathbf{x}_{i j} \mid \omega_{\text {unk }}\right), \forall \mathbf{x}_{i j} \in \mathrm{X}$. The proposed approach is based on the observation that the PDF of each pixel can be modeled as a mixture of the conditional PDFs of both the classes of interest and the unknown class:

$$
p\left(\mathbf{x}_{i j}\right)=\sum_{s=1}^{S} P\left(\omega_{\mathrm{nts}}\right) \cdot p\left(\mathbf{x}_{i j} \mid \omega_{\mathrm{nts}}\right)+P\left(\omega_{\mathrm{unk}}\right) \cdot p\left(\mathbf{x}_{i j} \mid \omega_{\mathrm{nnk}}\right)
$$

According to Parzen density estimation [1], we aim at obtaining a reliable nonparametric estimate for $p\left(\mathbf{x}_{i j}\right)$ as a mixture of a suitable set of kernel functions $\Phi=\left\{\phi_{k}(\cdot)\right\}_{k=1}^{K}$ :

$$
\hat{p}\left(\mathbf{x}_{i j}\right)=\sum_{k=1}^{K} w_{k} \cdot \varphi_{k}\left(\mathbf{x}_{i j}\right)
$$

where $K$ denotes the number of kernels (a free parameter to be set by the user), and $\mathrm{W}=\left\{w_{k}\right\}_{k=1}^{K}$ represent the weights regulating the contribution of each kernel.

However, since the density $p\left(\mathbf{x}_{i j}\right)$ is given by a linear combination of $\left\{p\left(\mathbf{x}_{i j} \mid \omega_{\mathrm{nts} s}\right)\right\}_{s=1}^{S}$ and $p\left(\mathbf{x}_{i j} \mid \omega_{\text {unk }}\right)$, it is worth noting that, if the set of kernels $\Phi$ allows obtaining a reliable estimate $\hat{p}\left(\mathbf{x}_{i j}\right)$, then also the class-conditional densities can be reliably approximated as a linear combination of $\Phi$. Hence, they can be estimated as:

$$
\begin{aligned}
& \hat{p}\left(\mathbf{x}_{i j} \mid \omega_{\text {ints }}\right)=\sum_{k=1}^{K} w_{\text {ints } k} \cdot \phi_{k}\left(\mathbf{x}_{i j}\right) \\
& \hat{p}\left(\mathbf{x}_{i j} \mid \omega_{\text {unk }}\right)=\sum_{k=1}^{K} w_{\text {unk } k} \cdot \phi_{k}\left(\mathbf{x}_{i j}\right)
\end{aligned}
$$

where $\mathrm{W}_{\text {ints }}=\left\{w_{\text {ints } k}\right\}_{k=1}^{K}, \mathrm{~W}_{\text {unk }}=\left\{w_{\text {unk } k}\right\}_{k=1}^{K}$ are the weights regulating the contribution of each kernel for the $s$-th class of interest and the unknown class, respectively.

As commonly done in the literature, we consider normalized isotropic Gaussian kernels, i.e. 


$$
\phi_{k}\left(\mathbf{x}_{i j}\right)=\frac{1}{\left(\sqrt{2 \pi \sigma_{k}^{2}}\right)^{D}} \exp \left[-\frac{\left\|\mathbf{x}_{i j}-\boldsymbol{\mu}_{k}\right\|^{2}}{2 \sigma_{k}^{2}}\right]
$$

where $\boldsymbol{\mu}_{k}$ is the centre and $\sigma_{k}^{2}$ is the variance (which tunes the smoothness of the estimate).

In the following, we describe into details the procedures adopted for estimating $\hat{p}\left(\mathbf{x}_{i j}\right)$, $\hat{p}\left(\mathbf{x}_{i j} \mid \omega_{\mathrm{nts}}\right)$ and $\hat{p}\left(\mathbf{x}_{i j} \mid \omega_{\mathrm{unk}}\right)$, respectively.

\subsubsection{Estimation of $\hat{p}\left(\mathbf{x}_{i j}\right)$}

For computing both the centers $M=\left\{\boldsymbol{\mu}_{k}\right\}_{k=1}^{K}$ and variances $\Sigma=\left\{\sigma_{k}^{2}\right\}_{k=1}^{K}$ of all the kernels, as well as the set of weights $\mathrm{W}$ defining $\hat{p}\left(\mathbf{x}_{i j}\right)$, we employ the EM algorithm over all the pixels of $\mathrm{X}$. EM allows determining the maximum likelihood (ML) estimator of the parameters characterizing a certain distribution in the presence of incomplete observations [2]. Indeed, our objective is to identify the ML estimate for the set of parameters $\theta=\{M, \Sigma, \mathrm{W}\}=\left\{\boldsymbol{\mu}_{k}, \sigma_{k}^{2}, w_{k}\right\}_{k=1}^{K}$ that allows maximizing the log-likelihood of $\mathrm{X}$, i.e.

$$
\ln \mathrm{L}(\theta)=\ln [p(\mathrm{X} \mid \theta)]=\sum_{i, j=1}^{I, J} \ln \left[\hat{p}\left(\mathbf{x}_{i j}\right)\right]
$$

At each iteration $l$, the set of estimated parameters $[\theta]^{(l)}$ provides an increase in the loglikelihood until a local maximum is reached, i.e. $\ln \mathrm{L}\left([\theta]^{(l)}\right) \geq \ln \mathrm{L}\left([\theta]^{(l)}\right)$.

For simplicity, weights are initially set to $1 / K$, whereas kernel parameters are initialized using the $k$-means clustering algorithm [7] fixing the number of clusters equal to $K$. In particular, centers and variances of the Gaussians are initialized to the centers and variances of the resulting clusters. Then, according to [2] the updated estimates for the unknown parameters are given by:

$$
\begin{gathered}
{\left[w_{k}\right]^{(l+1)}=\frac{\sum_{i=1}^{I} \sum_{j=1}^{J} \frac{\left[w_{k}\right]^{(l)} \cdot\left[\phi_{k}\left(\mathbf{x}_{i j}\right)\right]^{(l)}}{\left[\hat{p}\left(\mathbf{x}_{i j}\right)\right]^{(l)}}}{I \cdot J}} \\
{\left[\boldsymbol{\mu}_{k}\right]^{(l+1)}=\frac{\sum_{i=1}^{I} \sum_{j=1}^{J} \frac{\left[w_{k}\right]^{(l)} \cdot\left[\phi_{k}\left(\mathbf{x}_{i j}\right)\right]^{(l)}}{\left[\hat{p}\left(\mathbf{x}_{i j}\right)\right]^{(l)}} \cdot \mathbf{x}_{i j}}{\sum_{i=1}^{I} \sum_{j=1}^{J} \frac{\left[w_{k}\right]^{(l)} \cdot\left[\phi_{k}\left(\mathbf{x}_{i j}\right)\right]^{(l)}}{\left[\hat{p}\left(\mathbf{x}_{i j}\right)\right]^{(l)}}}} \\
{\left[\sigma_{k}^{2}\right]^{(l+1)}=\frac{\sum_{i=1}^{I} \sum_{j=1}^{J} \frac{\left[w_{k}\right]^{(l)} \cdot\left[\phi_{k}\left(\mathbf{x}_{i j}\right)\right]^{(l)}}{\left[\hat{p}\left(\mathbf{x}_{i j}\right)\right]^{(l)}} \cdot\left(\mathbf{x}_{i j}-\left[\boldsymbol{\mu}_{k}\right]^{(l+1)}\right)}{D \cdot \sum_{i=1}^{I} \sum_{j=1}^{J} \frac{\left[w_{k}\right]^{(l)} \cdot\left[\phi_{k}\left(\mathbf{x}_{i j}\right)\right]^{(l)}}{\left[\hat{p}\left(\mathbf{x}_{i j}\right)\right]^{(l)}}}}
\end{gathered}
$$

Reasonably, we assume that convergence is reached when the relative increase in the log-likelihood is lower than a prefixed threshold $\varepsilon$.

\subsubsection{Estimation of $\hat{p}\left(\mathbf{x}_{i j} \mid \omega_{\mathrm{nts}}\right)$}

Once $M$ and $\Sigma$ have been determined (and hence the set of kernels $\Phi$ has been properly defined), we exploit the available ground truth for the $s$-th class of interest $\omega_{\mathrm{nts}}$ for deriving the estimate of the corresponding conditional density $\hat{p}\left(\mathbf{x}_{i j} \mid \omega_{\text {nts }}\right)$. In particular, the set of weights $\mathrm{W}_{\text {ints }}$ associated to $\omega_{\mathrm{nts}}$ is determined using again the EM algorithm, but solely on the available training samples $\mathrm{T}_{s}=\left\{\mathbf{x}_{i j} \in \mathrm{X} \mid y_{i j}=\omega_{\mathrm{nts}}\right\},\left|\mathrm{T}_{s}\right|=N_{s}$, where $y_{i j}$ denotes the true label for pixel at position $(i, j)$. 
Weights are initialized to $1 / K$, and then updated (according to [2]) using the following equation:

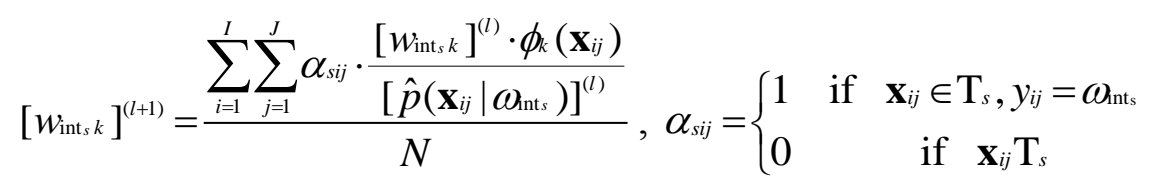

The corresponding log-likelihood is given by:

$$
\ln \mathrm{L}\left(\theta \equiv \mathrm{W}_{\mathrm{ints}}\right)=\sum_{i, j=1}^{I, J} \alpha_{s i j} \cdot \ln \left[\hat{p}\left(\mathbf{x}_{i j} \mid \omega_{\mathrm{ints}}\right)\right]
$$

Even in this case we assume that convergence is reached if the relative increase in the loglikelihood is lower than $\varepsilon$.

\subsubsection{Estimation of $\hat{p}\left(\mathbf{x}_{i j} \mid \omega_{\mathrm{nnts}}\right)$}

In accordance with (3), being $P\left(\omega_{\mathrm{mk}}\right)=1-\sum P\left(\omega_{\mathrm{mt}}\right)$, the conditional density of the unknown class can be estimated (provided that we can obtain a reliable estimate $\left\{\hat{P}\left(\omega_{\mathrm{nt} s}\right)\right\}_{s=1}^{S}$ for the prior probabilities of the classes of interest) as follows:

$$
\hat{p}\left(\mathbf{x}_{i j} \mid \omega_{\text {unk }}\right)=\frac{\sum_{k=1}^{K} w_{k} \cdot \varphi_{k}\left(\mathbf{x}_{i j}\right)-\sum_{s=1}^{S} \hat{P}\left(\omega_{\mathrm{int}_{s}}\right) \cdot \sum_{k=1}^{K} w_{\mathrm{int}_{s} k} \cdot \varphi_{k}\left(\mathbf{x}_{i j}\right)}{1-\sum_{s=1}^{S} \hat{P}\left(\omega_{\mathrm{int}_{s}}\right)}
$$

A first rough estimate $\left[\hat{p}\left(\mathbf{x}_{i j} \mid \omega_{\text {unk }}\right)\right]^{(0)}$ can be obtained either simply fixing $\left[\hat{P}\left(\omega_{\text {int }_{s}}\right)\right]^{(0)}=1 /(S+1)$ or through the employment of the significance testing approach [8]. Both the quantities are then iteratively updated in the following phase.

According to (14), it is worth noting that an acceptable solution to explicitly determine $\mathrm{W}_{\mathrm{unk}}$ is given by:

$$
w_{\text {unk } k}=\frac{w_{k}-\sum_{s=1}^{S} \hat{P}\left(\omega_{\mathrm{int}_{s}}\right) \cdot w_{\text {int }_{s} k}}{1-\sum_{s=1}^{S} \hat{P}\left(\omega_{\mathrm{int}_{s}}\right)}
$$

\subsection{Prior modeling}

For modeling the prior $P(\mathrm{C})$ we exploit the MRF technique [3]. In particular, we assume that the label $C_{i j}$ associated to the pixel at position $(i, j)$ depends on the labels associated to pixels belonging to the spatial neighborhood $\mathrm{G}_{i j}$ (we always considered first order neighborhoods).

In other words, the higher the number of a given pixel's spatial neighbors belonging to a certain land-cover class, the higher the probability that the given pixel itself belongs to the same class. In this hypothesis, it holds the equivalence:

$$
P\left(C_{i j} \mid C_{g h} ;(g, h) \in \mathrm{G}_{i j}\right)=Z^{-1} \cdot \exp \left[-U_{\text {contex }}\left(C_{i j}\right)\right]
$$

where $Z=Z\left(\mathrm{G}_{i j}\right)$ is a normalizing constant called partition function, while $U_{\text {contex }}$ is a Gibbs energy function (accounting for the spatial context) of the form: 


$$
U_{\text {contex }}\left(C_{i j}\right)=-\sum_{(g, h) \in \mathrm{G} i j} \beta \cdot \delta\left(C_{i j}, C_{g h}\right)
$$

where $\beta>0$ tunes the influence of the context and $\delta$ is the Kronecker delta function defined as:

$$
\delta\left(C_{i j}, C_{g h}\right)=\left\{\begin{array}{lll}
1 & \text { if } & C_{i j}=C_{g h} \\
0 & \text { if } & C_{i j} \neq C_{g h}
\end{array}\right.
$$

\section{Iterative labeling}

Solving Eq. (2) is equivalent to maximize the $\log$-likelihood $\ln \mathrm{L}(\mathrm{X} \mid \mathrm{C})$, which can be written as:

$$
\ln \mathrm{L}(\mathrm{X} \mid \mathrm{C})=-U_{\text {data }}(\mathrm{X}, \mathrm{C})-U_{\text {contex }}(\mathrm{C})-\ln (Z)
$$

where $U_{\text {data }}(\mathrm{X}, \mathrm{C})=-\ln [p(\mathrm{X} \mid \mathrm{C})]$, represents the class-conditional energy function while $U_{\text {contex }}$ is given by (17). Since $Z$ solely depends on the selected type of neighborhood, the final problem becomes solving:

$$
\tilde{\mathrm{C}}=\underset{\mathrm{C}}{\operatorname{argmin}}\left\{U_{\text {contex }}(\mathrm{C})+U_{\text {data }}(\mathrm{X}, \mathrm{C})\right\}
$$

To this aim, we propose a strategy based on the Iterated Conditional Modes (ICM) algorithm [9] which allows maximizing local conditional probabilities sequentially. In particular, at each iteration $l$ we update the estimated prior probabilities for the classes of interest $\left\{\hat{P}\left(\omega_{\mathrm{nts}}\right)\right\}_{s=1}^{S}$ and, accordingly, also the class-conditional density of the unknown class $\hat{p}\left(\mathbf{x}_{i j} \mid \omega_{\text {unk }}\right)$. The algorithm works as follows:

Step 1. After estimating $\left\{\hat{p}\left(\mathbf{x}_{i j} \mid \omega_{\mathrm{nts}}\right)\right\}_{s=1}^{S}, \forall \mathbf{x}_{i j} \in \mathrm{X}$, following the approach described in the previous paragraphs, set $\left\{\hat{P}\left(\omega_{\mathrm{nts}}\right)\right\}=1 /(S+1)$ (no prior knowledge is assumed to be available about the true prior $\left.P\left(\omega_{\text {nts }}\right)\right)$ and compute the conditional density of the unknown class $\hat{p}\left(\mathbf{x}_{i j} \mid \omega_{\text {unk }}\right)$ accordingly;

Step 2. Derive the initial sets of labels $\tilde{\mathrm{C}}$ by solely minimizing the non-contextual term of Eq. (20), i.e. $\tilde{\mathrm{C}}=\arg \min \left\{U_{\text {data }}(\mathrm{X}, \mathrm{C})\right\}$;

Step 3. On the basis of current $\tilde{\mathrm{C}}$, compute the new estimated prior probabilities ratioing the number of pixels associated to the corresponding class of interest over the whole number of pixels, i.e. $\hat{P}\left(\omega_{\mathrm{nts}}\right)=\left|\tilde{\mathrm{G}}_{\mathrm{nts}}\right| /(I \cdot J), \quad \tilde{\mathrm{C}}_{\mathrm{nts}}=\left\{C_{i j} \mid C_{i j} \in \tilde{\mathrm{C}}, C_{i j}=\omega_{\mathrm{nts}}\right\}_{i, j=1}^{I, J} ;$ then, update the classconditional densities for the unknown class $\hat{p}\left(\mathbf{x}_{i j} \mid \omega_{\mathrm{unk}}\right)$ accordingly;

Step 4. Update $\tilde{\mathrm{C}}$ according to Eq. (20);

Step 5. Repeat Step 3 and Step 4 until no changes occur between successive iterations.

\section{Experimental results}

In order to assess the effectiveness of the proposed technique, we carried out several experiments with different combinations of multispectral, hyperspectral and SAR data. Here we focus the attention on a representative targeted land-cover classification problem referring to an intense farming district located in Barrax, a village close to Albacete (Castilla-La Mancha, Spain). In particular, we considered a hyperspectral PROBA CHRIS image (composed by 63 spectral bands with centre wavelengths from 400 to $1050 \mathrm{~nm}$ ) acquired on $16^{\text {th }}$ July 2004 and identified a study area of $512 \times 512$ pixels (see Fig. 1.a). 
Available prior knowledge has been used for defining regions of interest for all the 9 land-cover classes characterizing the study area (i.e., alfalfa, corn, garlic, grasslands, onions, potatoes, spring crops, stubble and sunflowers). Then, we split all the available labeled patterns into a training set and a validation set composed by 10361 and 11580 samples, respectively (see Table 1).

Table 1. Number of labeled samples available for each land-cover class characterizing the study area.

\begin{tabular}{|c|c|c|}
\hline Land-cover class & Training set & Validation set \\
\hline Alfalfa & 836 & 634 \\
\hline Corn & 2664 & 2481 \\
\hline Garlic & 302 & 167 \\
\hline Grasslands & 42 & 46 \\
\hline Onions & 220 & 150 \\
\hline Potatoes & 283 & 195 \\
\hline Spring crops & 3318 & 5165 \\
\hline Stubble & 2247 & 1900 \\
\hline Sunflowers & 449 & 842 \\
\hline Total & 10361 & 11580 \\
\hline
\end{tabular}

In our trials we focused on four land-cover classes, namely alfalfa, corn, garlic and potatoes. In particular, we carried out experiments over all corresponding 15 combinations obtained varying $S$ from 1 to 4 . It is worth noting that only the labeled patterns of the training set available for the specific classes of interest considered in each case were used while running the proposed technique. Moreover, classification accuracies were evaluated over the samples of the validation set by considering each time all the non-interest classes as belonging to the unknown class.

As described in Section 3, the user is required to set the number of Gaussian kernels $K$ to be employed for approximating the PDFs. Hence, in order to understand how significant the selection of this free parameter is, we performed a series of experiments setting $K=A \cdot(S+1)$ and varying $A$ from 10 to 40 with steps of 5 . Moreover, according to a variety of experiments on toy and real datasets we fixed $\varepsilon=10^{-4}$ and $\beta=10^{2}$.

In all the trials we employed the $k$-means clustering for initializing both centers and variances of kernel functions. Nevertheless, the very high complexity of the algorithm (i.e., approximately $O\left(N^{D(K+1)} \log N\right)$, where $N$ and $D$ represent the number of samples to be clustered and their dimensionality, respectively [10]), prevented us from using all the patterns of the investigated image, as this would have required a very high computational burden. In order to overcome this limitation, we ran the $k$-means algorithm on a random subset containing one third of the total amount of samples. However, as this might affect the final classification accuracies of the proposed technique, for each value of $A$, we performed 10 different trials running each time the $k$-means clustering on a different random subset. Moreover, we finally also combined the resulting targeted land-cover maps through a majority voting ensemble (denoted as $\mathrm{TC}_{M V}$ ).

For validating the potentialities of the presented method, we compared the results with those obtained by:

- Fully-supervised SVM classifiers trained by using all the available labeled samples of the training set [for the selection of the two free parameters (i.e., a penalization parameter and the variance of considered Gaussian kernels) we employed a 10-fold cross-validation strategy [1]]

- Ensembles of one-class SVDD classifiers associated to each specific targeted land-cover class of interest [for the selection of the free parameters (i.e., the expected percentage of 
outliers in the training set and the variance of considered Gaussian kernels) we employed the same strategy adopted in [11]].

Classification accuracies have been evaluated in terms of kappa coefficient, which allows to take into consideration errors and their type [6].

In Table 2 we show the results obtained with the proposed technique for $K=10,20,30,40$. In particular, we report the median over the 10 realizations with different $k$-means clustering initialization. Moreover, also accuracies finally obtained with the majority voting ensemble $\mathrm{TC}_{M V}$, as well as those obtained by the SVDD ensembles and fully-supervised SVM are presented.

Table 2. Kappa coefficient of accuracy obtained for all the 15 investigated targeted land-cover classification problems.

\begin{tabular}{|c|c|c|c|c|c|c|c|c|c|c|c|}
\hline \multirow{2}{*}{$S$} & \multirow{2}{*}{ Alfalfa } & \multirow{2}{*}{ Corn } & \multirow{2}{*}{ Garlic } & \multirow{2}{*}{ Potatoes } & \multicolumn{4}{|c|}{$A$} & \multirow{2}{*}{$\mathrm{TC}_{M V}$} & \multirow{2}{*}{ SVDD } & \multirow{2}{*}{ SVM } \\
\hline & & & & & 10 & 20 & 30 & 40 & & & \\
\hline 4 & $\overline{\theta \otimes}$ & $\otimes$ & $\otimes$ & $\otimes$ & $\overline{c 0.9409}$ & 0.9050 & 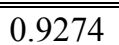 & $\begin{array}{l}0.9216 \\
\end{array}$ & 0.9395 & 0.8618 & 0.9509 \\
\hline \multirow{4}{*}{3} & $\otimes$ & $\otimes$ & $\otimes$ & & 0.9408 & $\begin{array}{l}0.9334 \\
\end{array}$ & 0.9500 & $\begin{array}{l}0.9437 \\
\end{array}$ & 0.9580 & 0.8567 & 0.9620 \\
\hline & $\otimes$ & $\otimes$ & & $\otimes$ & 0.9552 & 0.9460 & 0.9432 & 0.9234 & 0.9478 & 0.9292 & 0.9625 \\
\hline & $\otimes$ & & $\otimes$ & $\otimes$ & 0.8555 & 0.8676 & 0.8236 & 0.7799 & 0.8481 & 0.6808 & 0.9060 \\
\hline & & $\otimes$ & $\otimes$ & $\otimes$ & 0.8658 & 0.9533 & 0.9527 & 0.9446 & 0.9586 & 0.8436 & 0.9414 \\
\hline \multirow{6}{*}{2} & $\bar{\otimes}$ & $\otimes$ & & & 0.9465 & 0.9529 & 0.9593 & 0.9600 & 0.9642 & 0.9275 & 0.9747 \\
\hline & $\otimes$ & & $\otimes$ & & 0.7349 & 0.7844 & 0.8327 & 0.8751 & 0.8579 & 0.6213 & 0.9329 \\
\hline & $\otimes$ & & & $\otimes$ & 0.8862 & 0.8664 & 0.8566 & 0.8154 & 0.8594 & 0.8231 & 0.9342 \\
\hline & & $\otimes$ & $\otimes$ & & 0.9405 & 0.9631 & 0.9679 & 0.9748 & 0.9704 & 0.8807 & 0.9541 \\
\hline & & $\otimes$ & & $\otimes$ & 0.8681 & 0.9650 & 0.9328 & 0.9612 & 0.9686 & 0.9195 & 0.9547 \\
\hline & & & $\otimes$ & $\otimes$ & 0.6527 & 0.8410 & 0.7129 & 0.6952 & 0.8075 & 0.4648 & 0.7858 \\
\hline \multirow{4}{*}{1} & $\otimes$ & & & & 0.8235 & 0.8790 & 0.8906 & 0.8900 & 0.9176 & 0.7761 & 0.9753 \\
\hline & & $\otimes$ & & & 0.9029 & 0.9806 & 0.9830 & 0.9830 & 0.9820 & 0.9696 & 0.9690 \\
\hline & & & $\otimes$ & & 0.3825 & 0.3975 & 0.4424 & 0.4696 & 0.4304 & 0.3576 & 0.7887 \\
\hline & & & & $\otimes$ & 0.4174 & 0.7461 & 0.7512 & 0.7340 & 0.7702 & 0.5950 & 0.7860 \\
\hline
\end{tabular}

From Table 2, we can notice that the proposed method proved generally rather stable versus different values of $A$ (only for $A=10$ we experienced lower performances in some few cases) and always outperforms the approach based on the SVDD ensembles. In particular, by comparing the classification accuracies with those obtained with the majority voting ensemble $\mathrm{TC}_{M V}$ the gap in terms of kappa coefficient is on average higher than 0.11 with peaks corresponding to the targeted classification problems with $S=2$ where the land-cover classes of interest are garlic-potatoes (i.e., 0.3427) and alfalfa-garlic (i.e., 0.2366), respectively. Moreover, it is particularly interesting to note that also when a single class of interest is considered (this corresponds to the case which the SVDD classifiers have been specifically designed for) the proposed TC method still exhibits better performances, even in the critical cases where the reduced amount of labeled samples for the investigated class (i.e., garlic and potatoes) does not allow to obtain high classification accuracies.

However, the most remarkable observations arise when comparing the results with those obtained by fully-supervised SVM. Indeed, classification accuracies are very close to the ones exhibited by the proposed TC technique (i.e., the average gap in terms of kappa is lower than 0.04), despite solely labeled samples for the only classes of interest are exploited. This behavior is also confirmed by qualitatively comparing final targeted land-cover map, which look very similar to each other (see for instance Fig. 1 where maps for the case corresponding to $S=4$ are reported), thus confirming the effectiveness of the presented method. 


\section{Conclusions}

In this paper we presented a novel targeted classification (TC) technique capable of addressing problems where the objective is to identify one (or few) land-cover classes of interest, under the assumption that ground-truth information is available for the only (few) class(es) of interests.

In this context, fully-supervised approaches cannot be effectively employed due to the absence of a complete and exhaustive ground truth. The proposed method, instead, allows exploiting the only prior knowledge available for the specific land-cover classes of interest, while providing accuracies comparable with those of fully-supervised methods. In particular, the TC technique jointly exploits the Expectation-Maximization (EM) algorithm and an iterative labelling strategy based on Markov random fields (MRF) accounting for spatial correlation. Experimental results on a challenging datasets derived from hyperspectral CHRIS PROBA data confirmed the effectiveness and the reliability of the proposed technique.

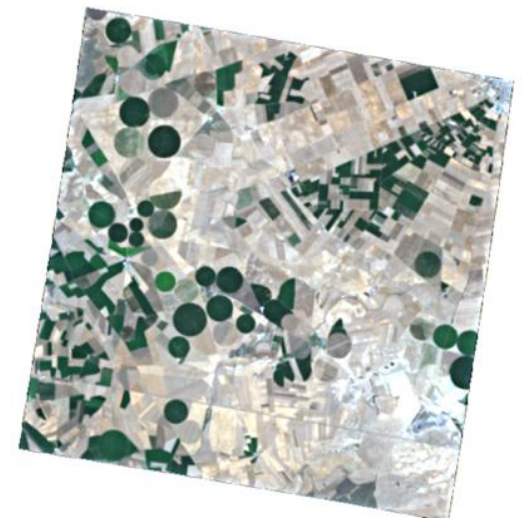

(a)

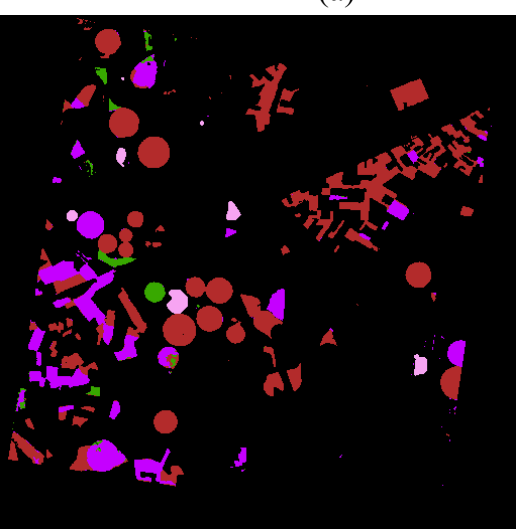

(c)

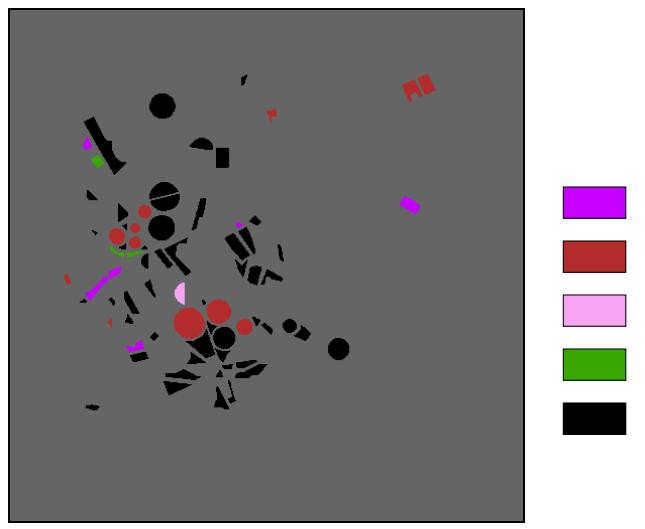

alfalfa

corn

garlic

potatoes

unknown

(b)

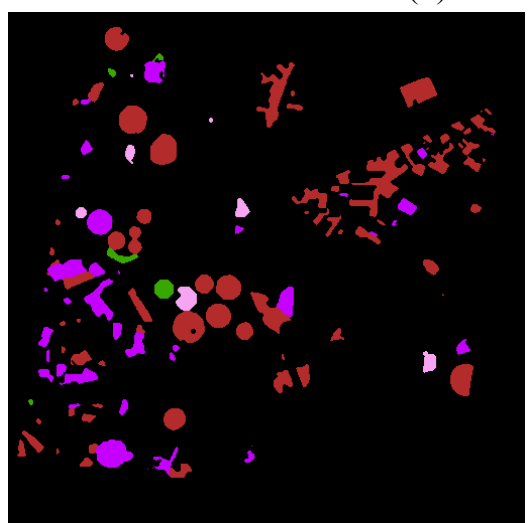

(d)

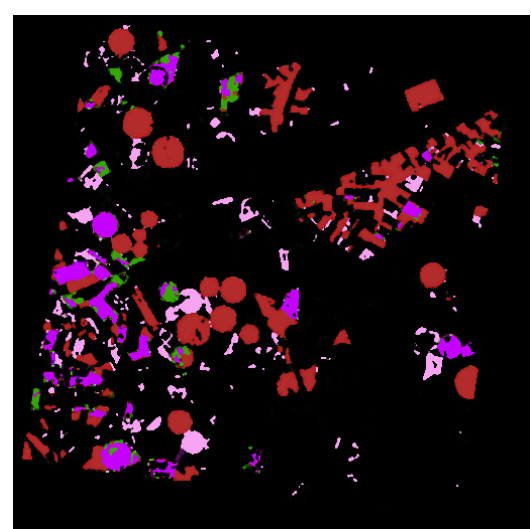

(e)

Figure 1: (a) RGB composition [band 23 (red), band 14 (green), band 7 (blue)] of the investigated PROBA CHRIS image. Targeted land-cover classification problem with four classes of interest (i.e., alfalfa, corn, garlic, potatoes): (b) available ground truth; land-cover maps obtained by: (c) fully supervised SVM; (d) the proposed targeted classifier with the majority voting ensemble $\mathrm{TC}_{\mathrm{MV}}$; (e) the ensemble of SVDD classifiers.

\section{References}

[1] Duda, R. O., Hart, P. E., and Stork, D. G., 2000. Pattern Classification. Wiley, New York.

[2] Dempster, A., Laird, N., and Rubin, D., 1977. Maximum Likelihood from Incomplete Data Via the EM Algorithm. The Royal Statistical Society, Series B, 39(1), pp. 1-38.

[3] Solberg, A. H. S., Taxt, T., and Jain, A. K., 1996. A Markov Random Field Model for Classification of Multisource Satellite Imagery. IEEE Trans. Geosci. Remote Sens., 34(1), pp. 100-113. 
[4] Cristianini N., and Shawe-Taylor, J., 2000. An Introduction to Support Vector Machines. Cambridge University.

[5] Tax, D. M. J., and Duin, R. P. W., 2004. Support vector data description. Machine Learning. 54(1), pp.45-66.

[6] Richards, J. A., and Jia, X., 2006. Remote Sensing Digital Image Analysis. An Introduction. Springer, Berlin.

[7] Bruzzone, L., and Fernàndez-Prieto, D., 1999. A Technique for the Selection of Kernel-Function Parameters in RBF Neural Networks for Classification of Remote-Sensing Images. IEEE Trans. Geosci. Remote Sens., 37(2), pp. 1179-1184.

[8] Jeon, B., and Landgrebe, D. A., 1999. Partially supervised classification using weighted unsupervised clustering. IEEE Trans. Geosci. Remote Sens., 37(3), pp. 1073-1079.

[9] Besag, J., 1986. On the statistical analysis of dirty pictures. Journal of the Royal Statistical Society, Series B, 48, pp. 259-302.

[10] Inaba, M., Katoh, N., and Imai, H., 1994. Applications of weighted voronoi diagrams and randomization to variance-based k-clustering. Proceedings of the Annual Symposium on Computational Geometry, pp. 332-339.

[11] Muñoz-Marí, J., Bovolo, F., Gómez-Chova, L., Bruzzone, L., and Camps-Valls, G., 2010, Semisupervised One-Class Support Vector Machines for Classification of Remote Sensing Data. IEEE Trans. Geosci. Remote Sens., 48(8), pp. 3188-3197. 